- Ann. Biol. anim. Bioch. Biophys., I962, 2 (4), 299-3 I7.

\title{
EVOLUTION DE LA COMPOSITION CHIMIQUE DU COLOSTRUM CHEZ LA TRUIE
}

\author{
E. SALMON-LEGAGNEUR et L. GUEGUEN \\ avec la collaboration technique de J. RetTagliatr, \\ Colette Baudrillart et Michelle Frasnier-Langlois
}

Station de Recherches sur l'Élevage des Porcins et Station centrale de Recherches de Nutrition, Centre national de Recherches zotechniques, Jouy-en Josas (Seine et Oise)

\section{SOMMAIRE}

L'étude de l'évolution des principaux constituants chimiques de la sécrétion des premiers jours de lactation (eau, lactose, matière grasse, azote total, fractions azotées, $\mathrm{P}, \mathrm{Ca}, \mathrm{K}, \mathrm{Na}, \mathrm{Mg}$ ) a été entreprise chez la Truie.

L'évolution des teneurs de la plupart des constituants est analogue à celle rencontrée chez les autres espèces, sauf pour les taux de matière grasse, de phosphore, de calcium et d'azote non protéique. Ces phénomènes, ainsi que les relations entre certains constituants, sont discutés.

\section{IN'TRODUC'TION}

Si l'on dispose maintenant d'un certain nombre d'études sur la composition du lait de Truie et son évolution au cours de la lactation, les renseignements sont, par contre, moins nombreux, ou plus inégaux sur la composition du colostrum de cette même espèce. Or, précisément, ce qui frappe dans les quelques travaux réalisés sur ce sujet, c'est la grande variabilité des résultats (tableau I), la rapidité de l'évolution de la composition (PERRIN, I954 - TRAVNICEK, I960) et les différences que l'on observe par rapport à certaines espèces, comme l'espèce bovine.

De ce fait, certaines questions sont restées jusqu'à présent sans réponse. Par exemple, si 1'on sait maintenant que la teneur maximum en lipides du lait de Truie se situe, non pas à 3 semaines comme on l'avait d'abord cru, mais dans les quelques heures qui suivent la parturition (SALMON-LEGAGNEUR, I959), on ignore toutefois à quel moment précis se situe ce maximum. De même, dans un travail précédent, nous n'avions pas élucidé pourquoi les teneurs des principaux minéraux paraissent suivre au début de la lactation chez la Truie une évolution très différente de celle observée chez la Vache (GuEguen et SALmon-I,EGaGneur, I959). 
E. SALMON-LEGAGNEUR, L. GUEGUEN

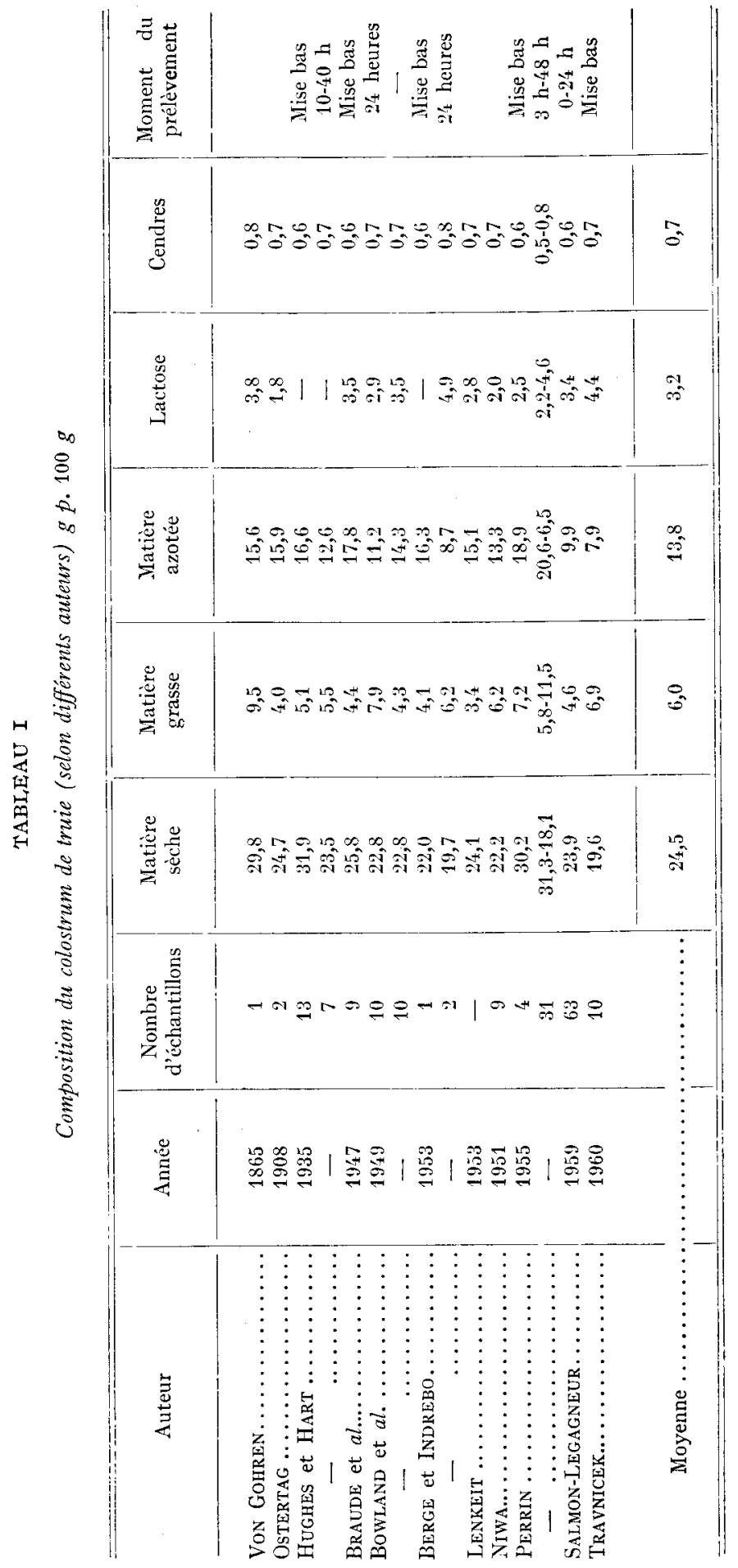


Nous avons pensé qu'une étude détaillée de la composition du liquide sécrété par la mamelle de la Truie au cours des quelques jours qui suivent la parturition pouvait nous éclairer sur certains points, en nous permettant, en particulier, de préciser l'évolution des principaux constituants de la sécrétion pendant cette période mal connue.

A cet effet, nous avons entrepris l'étude de la composition chimique d'une série d'échantillons de colostrum ( 192 au total) prélevés sur I6 truies de notre troupeau expérimental. L'analyse portait sur les principaux constituants (eau, lipides totaux, lactose, matière azotée totale, cendres), les éléments minéraux les plus importants (phosphore, calcium, sodium, potassium, magnésium) et sur diverses fractions azotées (caséine, albumine + globuline, proteose-peptone, azote non protéique.)

En outre, comme dans un travail précédent, nous avons cherché à savoir, par l'étude des corrélations, s'il existait des liaisons entre la sécrétion des différents constituants.

\section{MATÉRIEL E'T MÉTHODES}

$$
\text { A. - Animaux }
$$

Seize truies adultes de race Large White ayant déjà effectué une ou plusieurs portées furent utilisées : douze pour suivre l'évolution des principaux constituants et quatre pour l'évolution des fractions azotées.

Ces truies, fécondées au cours de l'automne I96I, étaient élevées en plein air au cours de la gestation et rentrées en cases individuelles en porcherie deux semaines avant la parturition. Pendant la gestation et la lactation, les truies recevaient une ration, calculée suivant les besoins, d'un aliment concentré équilibré.

\section{B. - Prélèvement des échantillons}

Les échantillons de sécrétion étaient prélevés sur chaque truie à des intervalles définis, en exprimant manuellement tout le liquide contenu dans les mamelles.

Sauf pour les deux premiers prélèvements, chaque récolte était précédée d'une injection intraveineuse de 2 unités internationales d'ocytocine pour provoquer l'éjection de la sécrétion. Cette injection était inutile pour les deux prélèvements effectués avant et au cours de la parturition, car, d'une part la quantité d'hormone posthypophysaire sécrétée par la truie à ce moment est suffisante pour que l'éjection ait lieu sans difficulté et que, d'autre part, une dose supplémentaire d'ocytocine à ce moment aurait pu perturber le déroulement normal de l'accouchement.

Le premier prélèvement était effectué en moyenne I à 2 heures avant la parturition, dès qu'il devenait possible de faire apparaître quelques gouttes de sécrétion en pinçant un trayon entre le pouce et l'index. Le second prélèvement avait lieu au moment de la naissance du premier porcelet, choisi comme temps zéro de la parturition.

Le prélèvement suivant correspondait à la fin de la parturition (rejet du placenta) et avait lieu en moyenne 6 heures après le temps zéro. Les prélèvements se succédaient ensuite de 6 heures en 6 heures jusqu'à 36 heures après le temps zéro, puis de $\mathbf{2} 2$ heures en 12 heures jusqu'à $7^{2}$ heures. Un dernier prélèvement était effectué à 96 heures, soit 4 jours après le temps zéro. Au total, 12 prélèvements par truie étaient donc pratiqués.

Le volume de chacun des échantillons récoltés variait suivant les animaux et les prélèvements de 50 à I $50 \mathrm{ml}$ environ. Il était en général plus élevé pour les derniers prélèvements que pour les premiers. Entre chaque prélèvement, les porcelets demeuraient avec leur mère et pouvaient donc téter librement. Compte tenu de l'irrégularité des tétées pendant cette période du début de lactation, aucune tentative de mesure des quantités secrétées ne fut entreprise.

Les échantillons issus d'une même truie étaient stockés sans conservateur, à o- $4^{\circ} \mathrm{C}$ à mesure de leur récolte. Ils étaient ensuite analysés par série dès que le dernier prélèvement avait été effectué. 


\section{C. - Méthodes d'analyse}

Les techniques utilisées pour le dosage des principaux constituants et des éléments minéraux sont celles que nous avons indiquées dans nos précédentes publications (E. Salmon-Legagneur, I959-GUEGUEN et JOURNET, I961), à savoir :

- Eau : dessiccation à $40^{\circ} \mathrm{C}$ pendant 48 heures, sous vide,

- Matière azotée totale : azote Kjeldahl $\times 6,38$,

- Lactose : dosage iodométrique du pouvoir réducteur,

- Lipides totaux : extraction éthéro-ammoniacale et évaporation,

- Matières minérales totales : incinération ¿̀ $55^{\circ} \mathrm{C}$.

- Phosphore : méthode colorimétrique au vanadomolybdate d'ammonium,

- Potassium, sodium, calcium, magnésium : spectrophotométrie de flamme.

Les différentes fractions azotées étaient dosées conventionnellement par leur azote après précipitation sélective. On procédait notamment aux déterminations suivantes :

I. - Azote total,

II. - Azote " non-caséine ", par précipitation de la caséine à pH 4,6 (Rowland, I938),

III. - Azote " non-caséine " moins azote " proteose-peptone ", par précipitation du filtrat précédent par le sulfate de soude en solution à I 2 p. Ioo (ASCHAFFEnBURG, I959),

IV. - Azote " non-protéique ", par précipitation à $0^{\circ} \mathrm{C}$ par l'acide trichloracétique à I 5 p. 100 (Shahani, 1951).

Par soustraction, on obtenait :

- Azote " caséine ": I - II,

- Azote " albumine + globuline " : III - IV,

- Azote "proteose-peptone ": II - III.

Les différents résultats étaient exprimés en grammes pour roo $\mathrm{g}$ du produit frais pour les principaux constituants, en grammes pour $\mathrm{I}$ ooo $\mathrm{g}$ pour les éléments minéraux et en milligrammes d'azote pour roo $\mathrm{g}$ pour les fractions azotées.

Dans l'exécution des calculs, les déterminations des valeurs moyennes par animal, ou par numéro de prélèvement, ont été faites à partir des résultats d'analyse sans correction pour la production laitière réelle qui n'était pas connue avec précision. Les valeurs moyennes indiquées sont donc des " moyennes non pondérées".

\section{RÉSULTATS}

\section{A. - Composition MOYENnE DU « COLOSTRUM »}

Par analogie avec les bovins, nous avons réservé conventionnellement l'appellation de "colostrum " à la secrétion des 24 heures postpartum (RowLAND, I953). Dans notre étude le colostrum est donc représenté par les prélèvements 2 à 6 inclus.

TABLEAU 2

Composition moyenne du colostrum de truie $(\mathrm{n}=\mathrm{I} 2)$

\begin{tabular}{|c|c|c|}
\hline Constituants & $\begin{array}{c}\text { Teneur moyenne } \\
\pm \sigma\end{array}$ & $\begin{array}{c}\text { Coefficient } \\
\text { de variation } \\
\text { interindividuel }\end{array}$ \\
\hline Eau p. $100 \ldots \ldots \ldots \ldots \ldots$ & $77,0 x \pm 1,95$ & 2,5 \\
\hline Matière sèche p. $100 \ldots \ldots \ldots \ldots$ & $22,96 \pm 1,95$ & 8,5 \\
\hline Matière azotée totale p. $100 \ldots \ldots$ & $13,82 \pm 1,80$ & 13,1 \\
\hline Matière grasse p. $100 \ldots \ldots \ldots \ldots$ & $4,70 \pm 1,76$ & 37,5 \\
\hline Lactose p. $100 \ldots \ldots \ldots \ldots \ldots$ & $3,59 \pm 0,33$ & 9,1 \\
\hline Matières minérales totales p. $100 \ldots$ & $0,72 \pm 0,05$ & 7,6 \\
\hline Phosphore p. $1000 \ldots \ldots \ldots \ldots \ldots$ & $1,14 \pm 0,10$ & 9,2 \\
\hline Calcium p. $1000 \ldots \ldots \ldots \ldots \ldots$ & $0,87 \pm 0,20$ & 22,7 \\
\hline Sodium p. $1000 \ldots \ldots \ldots \ldots \ldots$ & $0,65 \pm 0,15$ & 23,1 \\
\hline Potassium p. $1000 \ldots$ & $1,19 \pm 0,14$ & 11,1 \\
\hline Magnésium p. $1000 \ldots \ldots \ldots$ & $0,12 \pm 0,02$ & 16,7 \\
\hline
\end{tabular}


Nous avons calculé pour chaque truie la teneur moyenne du colostrum en ses principaux constituants et, à partir de ces résultats individuels, la moyenne et l'écarttype pour l'ensemble du lot, (tableau 2).

Ces résultats nous permettent de faire quelques commentaires :

I $^{0}$ Comme dans beaucoup d'espèces, la matière azotée est le constituant principal de la matière sèche du colostrum (60 p. Ioo). De leur côté, le phosphore et le potassium sont les éléments minéraux les plus abondants.

$2^{\circ}$ Par rapport au lait de Truie (cf. résultats antérieurs : SALMON-LEGaGneUR, I959), le colostrum est plus riche en matière azotée (augmentation de I37 p. IOo), en potassium ( + I9 p. IOo), en sodium ( $+86 \mathrm{p}$. IOo) et moins riche en matière grasse (diminution de 24 p. IOo), en lactose (-34 p. I00) en calcium (-60 p. roo) et en phosphore ( $-26 \mathrm{p}$. roo). On note aussi que le rapport calcium/phosphore est inférieur à I dans le colostrum, alors que dans le lait il y a plus de calcium que de phosphore.

$3^{\circ}$ Comme pour le lait, les variabilités individuelles des teneurs des principaux constituants ne sont pas identiques. Le constituant le plus variable est la matière grasse. Après l'eau, les constituants les moins variables sont le lactose et le phosphore. On retrouve ici la hiérarchie habituelle, identique à celle observée par WAITE et al. (I956) chez la Vache et par nous-mêmes (SALMON-LEGAGNEUR, I959) sur le lait de Truie.

\section{B. - ÉvOLUTION DES PRINCIPAUX CONSTITUANTS}

Les tableaux 3 et 4 et les figures I à 6 rapportent l'évolution de la composition de la sécrétion mammaire au cours des premiers jours de lactation.

TABLEAU 3

Éolution des teneurs des principaux constituants en pour 100

\begin{tabular}{|c|c|c|c|c|c|c|c|c|c|c|c|c|}
\hline No du prélèvement...$\ldots$ & 1 & 2 & 3 & 4 & 5 & 6 & 7 & 8 & 9 & 10 & 11 & 12 \\
\hline Stade de prélèvement .... & $\begin{array}{c}\text { avant } \\
\text { M } B .\end{array}$ & $0 \mathrm{~h}$ & $6 \mathrm{~h}$ & $12 \mathrm{~h}$ & $18 \mathrm{~h}$ & $24 \mathrm{~h}$ & $30 \mathrm{~h}$ & $36 \mathrm{~h}$ & $48 \mathrm{~h}$ & $60 \mathrm{~h}$ & $72 \mathrm{~h}$ & $4 \mathrm{j}$ \\
\hline Eau .... & 72,93 & 74,62 & 75,67 & 78,02 & 78,90 & 78,02 & 76,18 & 75,89 & 75,68 & 76,53 & 77,53 & 79,61 \\
\hline Matière sèche & 27,07 & 25,38 & 24,33 & 21,98 & 21,10 & 21,98 & 23,82 & 24,11 & 24,32 & 23,47 & 22,47 & 20,39 \\
\hline Matière azotée totale & 18,66 & 17,65 & 16,30 & 13,17 & 11,27 & 10,69 & 9,63 & 8,86 & 8,24 & 7,02 & 6,59 & 5,98 \\
\hline Matière grasse........ & 3,33 & 3,87 & 3,94 & 4,32 & 5,11 & 6,61 & 9,55 & 10,25 & 11,10 & 10,79 & 10,36 & 8,74 \\
\hline Lactose ......... & 3,24 & 3,21 & 3,31 & 3,68 & 3,92 & 3,87 & 3,90 & 4,01 & 4,22 & 4,63 & 4,71 & 4,86 \\
\hline Matières minérales totales. & 0,70 & 0,70 & 0,71 & 0,73 & 0,74 & 0,74 & 0,76 & 0,78 & 0,80 & 0,81 & 0,81 & 0,81 \\
\hline
\end{tabular}




\section{TABLEAU 4}

Évolution de la composition minérale en pour 1000

\begin{tabular}{|c|c|c|c|c|c|c|c|c|c|c|c|c|}
\hline Stade de prélèvement ... & $\begin{array}{l}\text { avant } \\
\text { M. B. }\end{array}$ & $0 \mathrm{~h}$ & $6 \mathrm{~h}$ & $12 \mathrm{~h}$ & $18 \mathrm{~h}$ & $24 \mathrm{~h}$ & $30 \mathrm{~h}$ & $36 \mathrm{~h}$ & $48 \mathrm{~h}$ & $60 \mathrm{~h}$ & $72 \mathrm{~h}$ & $96 \mathrm{~h}$ \\
\hline Phosphore & 1,08 & 1,08 & 1,09 & 1,12 & 1,20 & 1,22 & 1,31 & 1,32 & 1,36 & 1,37 & 1,39 & 1,40 \\
\hline Calcium & 0,69 & 0,71 & 0,75 & 0,85 & 0,96 & 1,01 & 1,17 & 1,30 & 1,42 & 1,56 & 1,61 & 1,62 \\
\hline Sodium . & 0,62 & 0,65 & 0,69 & 0,68 & 0,63 & 0,59 & 0,52 & 0,48 & $0,,^{\prime} \mathbf{t}^{\prime}$ & 0,35 & 0,34 & 0,32 \\
\hline Potassium & 1,27 & 1,25 & 1,19 & 1,17 & 1,20 & 1,16 & 1,16 & 1,14 & 1,14 & 1,12 & 1,09 & 1,09 \\
\hline Magnésium. & 0,114 & 0,111 & 0,122 & 0,123 & 0,123 & 0,118 & 0,120 & 0,122 & 0,124 & 0,115 & 0,114 & 0,103 \\
\hline
\end{tabular}

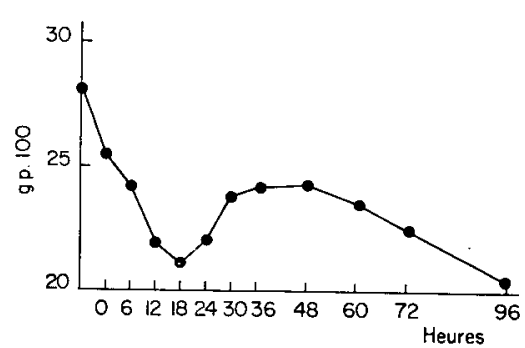

I. matière sèche

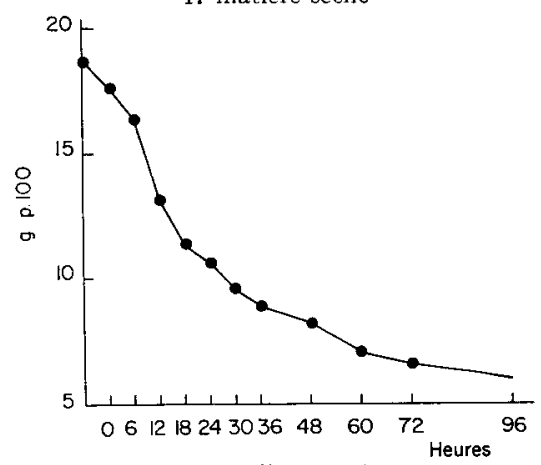

2. matière azotée

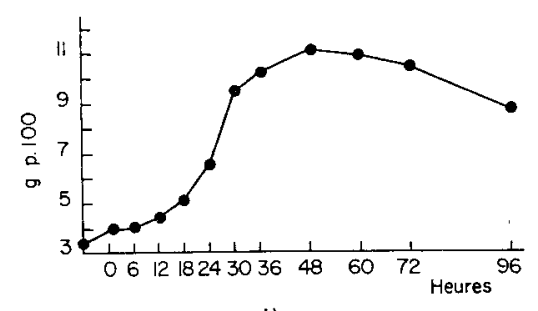

3. matière grasse

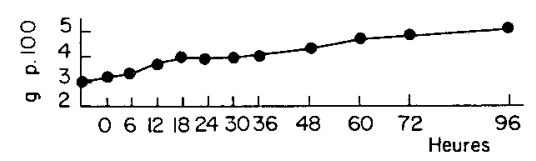

4. lactose

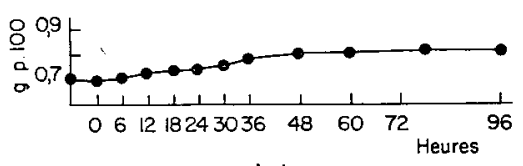

5. minéraux

FIG. I à 5. - Évolution des teneurs des principaux constituants 


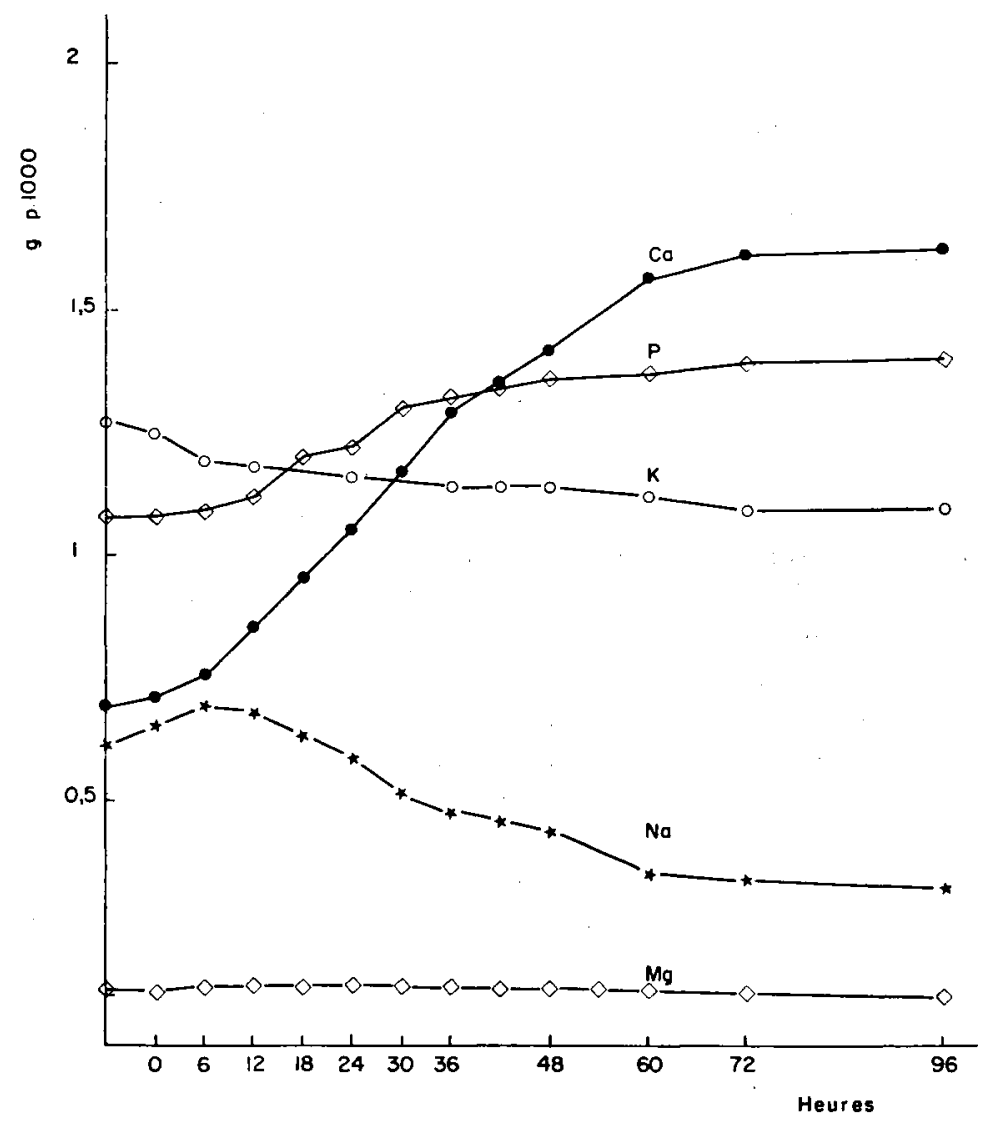

FIG. 6. - Évolution des teneurs des principaux élèments minéraux

Pour tous les constituants, l'évolution paraît importante et rapide, mais elle présente un caractère différent suivant chaque constituant :

\section{Io Eau et matière sèche}

Comme pour le lait, l'eau est en valeur relative le moins variable des constituants ( 9 p. Ioo pendant la période considérée). Toutefois, certaines sécrétions individuelles peuvent présenter des variations plus accusées, surtout si on les rapporte à la matière sèche. Dans notre expérience, les teneurs extrêmes en matière sèche ont été respectivement $22-30 \mathrm{p}$. Ioo pour le $\mathrm{I}^{\mathrm{er}}$ prélèvement et $\mathrm{I}_{7}-26 \mathrm{p}$. roo pour le dernier. Mais il est surtout curieux de constater que l'évolution de ce taux de matière sèche n'est pas régulière : elle présente successivement un minimum au bout de I 8 heures et un maximum au bout de 48 heures (Fig. I). Ce phénomène parait logique, car il résulte de l'évolution contraire des deux principaux constituants, la matière azotée et la matière grasse, dont les teneurs l'emportent tour à tour.

Annales de Biologie animale. - Ig62. 


\section{$2^{\circ}$ Matière azotée}

Entre la parturition et le $4^{\mathrm{e}}$ jour qui suit, la sécrétion s'appauvrit en azote d'environ $300 \mathrm{p}$. Ioo, ce qui est considérable, mais semble-t-il du même ordre que ce que l'on observe chez beaucoup d'espèces. Cette évolution se fait à une vitesse décroissante, suivant le phénomène que JoURNET et JARRIGE (I960) ont décrit pendant le premier mois de lactation chez la Vache.

\section{$3^{0}$ Matière grasse}

Initialement, pendant un peu moins de 24 heures, le taux de matière grasse est très bas et évolue assez lentement avec toutefois d'assez grandes variations individuelles (extrêmes au bout de I 8 heures : $2, \mathrm{I}-\mathrm{I0}, 5$ p. roo). Durant les 24 heures qui suivent, le taux s'accroît très rapidement et peut dépasser 16 p. Ioo pour certaines truies. Le moment exact où le taux maximum est atteint varie, selon les animaux, entre le $\mathrm{I}^{\mathrm{er}}$ et le $3^{\mathrm{e}}$ jour après la parturition.

Ceci explique parfaitement que, dans certaines études où le nombre de prélèvements était insuffisant, ce maximum ait pu souvent passer inaperçu. En outre, cela montre également que, pour une même date de prélèvement, on puisse rencontrer, suivant les animaux, des sécrétions très riches, ou relativement pauvres en lipides. Par exemple, les extrêmes à 48 heures sont 6,5 et i 6,7 p. Ioo.

Par la suite, le taux de matière grasse semble décroître assez régulièrement.

\section{$4^{\circ}$ Lactose et matières minérales}

Ces constituants présentent une évolution analogue, avec une augmentation régulière des teneurs au cours des jours qui suivent la parturition. Toutefois, ces augmentations n'ont pas la même valeur; elles sont respectivement de $66 \mathrm{p}$. roo pour le lactose et de I4 p. Ioo pour les matières minérales.

\section{$5^{\circ}$ Phosphore et calcium}

La teneur en phosphore augmente relativement lentement (30 p. Ioo en 4 jours) alors que la teneur en calcium augmente beaucoup plus pendant la même période (r30 p. I00). Ceci a pour conséquence qu'au bout de 48 heures environ, le taux de calcium devient supérieur au taux de phosphore.

\section{$6^{\circ}$ Potassium et sodium, magnésium}

Les teneurs en potassium et en sodium décroissent très sensiblement, respectivement de $\mathrm{I} 5 \mathrm{p}$. Ioo et de $50 \mathrm{p}$. Ioo. La légère augmentation de la teneur en sodium pendant les premières $\mathrm{I} 2$ heures n'est pas significative.

La teneur en magnésium est très faible et ne varie pas de façon apparente. 


\section{C. - Fractions azoTÉES}

La composition azotée moyenne du colostrum (sécrétion des premières 24 heures) pour les quatre truies étudiées est indiquée au tableau 5.

\section{TABLEAU 5}

Composition azotée du colostrum de Truie $(\mathrm{n}=4)$

\begin{tabular}{|c|c|c|}
\hline & mg d'azote $/ 100 \mathrm{~g}$ & P. 100 de l'azote \\
\hline Caséine..... & 724 & 31,8 \\
\hline Albumine + globuline .... & 1143 & 50,2 \\
\hline Protéose-peptone ...... & 341 & 14,9 \\
\hline Azote non protëique........ & 72 & 3,1 \\
\hline
\end{tabular}

L'évolution des différentes fractions au cours de l'ensemble de la période considérée est rapportée en valeurs absolue et relative aux tableaux 6 et 7 et aux figures 7 et 8 .

\section{TABLEAU 6}

Évolution de la teneur en azote ( $\mathrm{mg} / \mathrm{1} 00 \mathrm{~g}$. de secrétion)

\begin{tabular}{|c|c|c|c|c|c|c|c|c|c|c|c|c|}
\hline Prélèvements & $\begin{array}{l}\text { avant } \\
\text { M. B }\end{array}$ & $0 \mathrm{~h}$ & $6 \mathrm{~h}$ & $12 \mathrm{~h}$ & $18 \mathrm{~h}$ & $2^{\prime} \mathrm{h}$ & $30 \mathrm{~h}$ & $36 \mathrm{~h}$ & $48 \mathrm{~h}$ & $60 \mathrm{~h}$ & $72 \mathrm{~h}$ & $96 \mathrm{~h}$ \\
\hline Caséin & 838 & 795 & 796 & 724 & 650 & 650 & 663 & 652 & 563 & 549 & 544 & 531 \\
\hline Albumine + & 1689 & 1654 & 1389 & 1103 & 816 & 737 & 701 & 589 & 393 & 374 & 355 & 288 \\
\hline Protéose peptone......... & 595 & 517 & 480 & 288 & 227 & 187 & 122 & 158 & 82 & 66 & 59 & 52 \\
\hline Azote non protéique ...... & 65 & 67 & 69 & 70 & 76 & 80 & 85 & 85 & 88 & 87 & 90 & 88 \\
\hline Azote total ........ & 3187 & 3033 & 2734 & 2185 & 1775 & 1654 & 1571 & 1484 & 1126 & 1076 & 1048 & 959 \\
\hline
\end{tabular}

TABLEAU 7

Évolution des différentes fractions azotées en p. 100 de l'azote total

\begin{tabular}{|c|c|c|c|c|c|c|c|c|c|c|c|c|}
\hline Prélèvements & $\begin{array}{l}\text { avant } \\
\text { M. B }\end{array}$ & $0 \mathrm{~h}$ & $6 \mathrm{~h}$ & $12 \mathrm{~h}$ & $18 \mathrm{~h}$ & $2{ }^{\prime} \mathrm{h}$ & $30 \mathrm{~h}$ & $36 \mathrm{~h}$ & $48 \mathrm{~h}$ & $60 \mathrm{~h}$ & $72 \mathrm{~h}$ & $96 \mathrm{~h}$ \\
\hline Caséine & 26,3 & 26,2 & 29,1 & 33,1 & 36,9 & 39,3 & 42,2 & 43,9 & 50,0 & 51,0 & 52,0 & 55,5 \\
\hline Albumine + Globuline.... & 53,0 & $5 /, 5$ & 50,8 & 50,5 & 45,9 & 44,5 & 44,6 & 39,7 & 35,0 & 34,7 & 33,9 & 30,1 \\
\hline Protéose peptone........ & 18,7 & 17,0 & 17,6 & 13,2 & 12,8 & 11,3 & 7,8 & 10,6 & 7,2 & 6,1 & 5,6 & 5,4 \\
\hline Azote non protéique. & 2,0 & 2,2 & 2,5 & 3,2 & ${ }^{\prime}, 2$ & 4,8 & $5,4_{k}$ & 5,6 & 7,8 & 8,0 & 8,6 & 9,1 \\
\hline
\end{tabular}




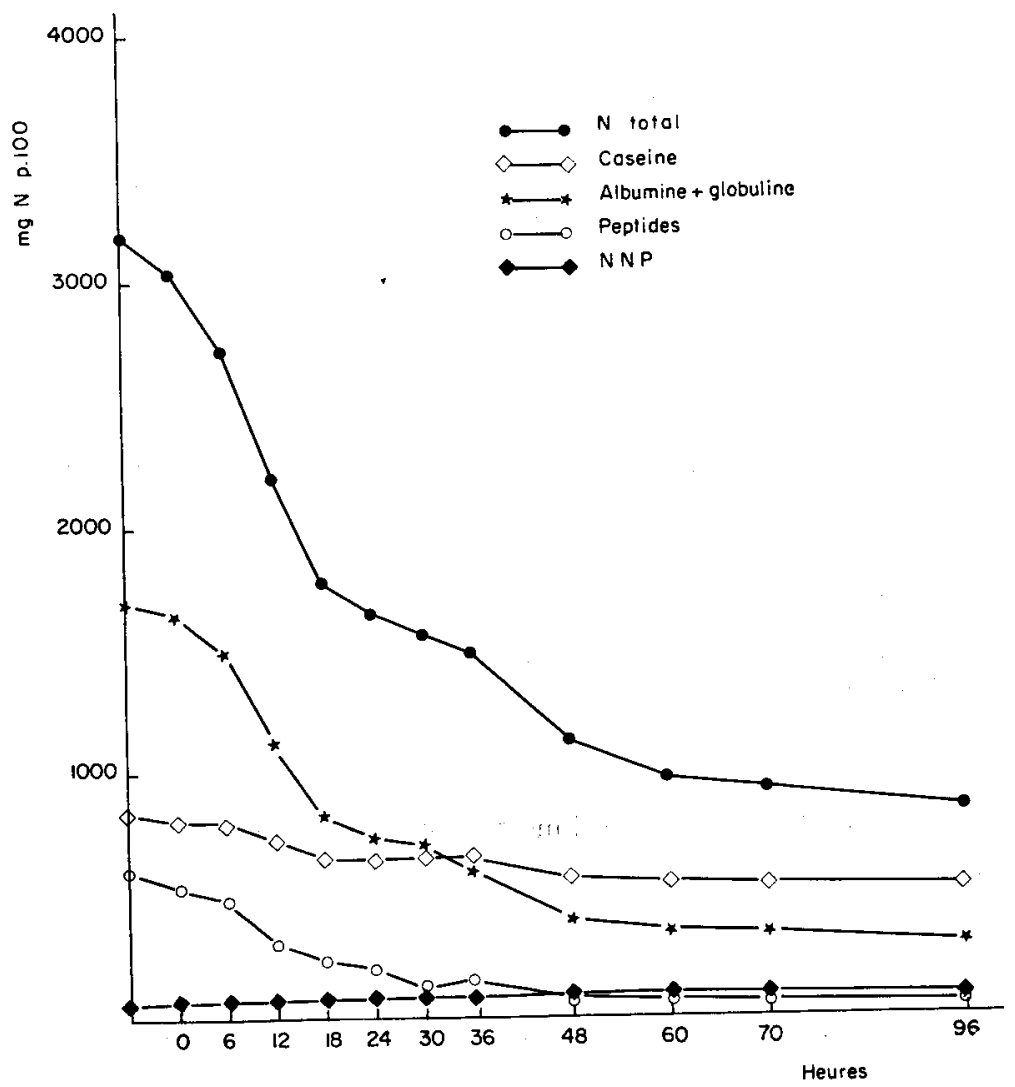

[iIG. 7. - Évolution des teneurs des principales fractions azotées ( $m g$ d'azote p. $100 \mathrm{~g}$ de sécrétion)

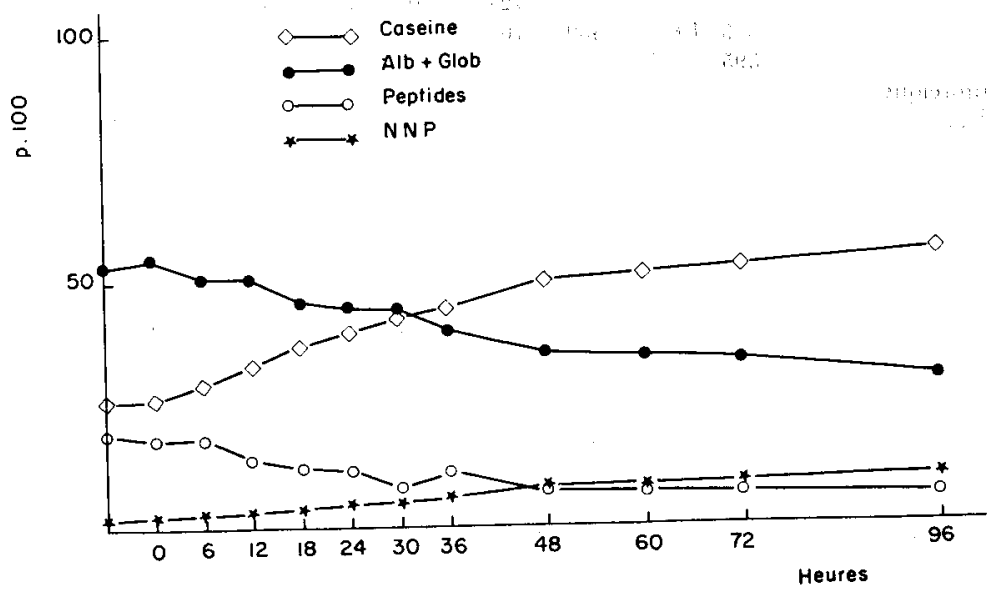

FIG. 8. - Évolution relative des différentes fractions azotées $g$ p. $100 \mathrm{~g}$ d'azote) 
On peut souligner les points suivants :

I $^{\mathrm{O}} \mathrm{La}$ fraction non protéique est la seule dont la teneur ait tendance à angmenter au cours du début de la lactation.

$2^{\circ}$ Parmi les protéines, ce sont les globulines, les albumines et les protéosepeptones qui diminuent le plus (80-92 p. I0o), alors que la caséine varie beaucoup moins ( 36 p. I00). Il s'en suit qu'à partir d'un certain moment, généralement au bout de 36 heures, la sécrétion de la caséine l'emporte sur celle des autres protéines, alors qu'auparavant les albumines et les globulines constituaient la fraction la plus importante.

\section{D. - RELATIONS ENTRE CONSTITUANTS}

Par analogie avec un travail antérieur sur le lait de Truie (SALMON-LEGAGNEUR, I 96 I), nous avons cherché à savoir, à l'aide du calcul de corrélations, s'il existait des liaisons entre certains constituants de la sécrétion et si ces liaisons étaient les mêmes que celles que nous avions trouvées pour le reste de la lactation. Toutefois, ne disposant pas de données suffisamment précises sur le volume de la sécrétion, nous avons dî nous limiter au calcul des corrélations totales, au lieu des corrélations partielles (indépendantes des quantités sécrétées) qui nous auraient apporté davantage de renseignements. Nos résultats devront donc être interprétés avec prudence.

\section{a) Relations intra-lactation}

Elles se rapportent aux évolutions relatives des constituants au cours de la période considérée. Nous avons trouvé entre les teneurs moyennes đe chaque constituant, au cours des différents prélèvements, les corrélations suivantes, choisies parmi les plus caractéristiques :

$$
\begin{aligned}
& \text { Lactose/natière azotée ............ } r=-0,96 \\
& \text { Lactose/matière grasse............ } r=+0,82 \\
& \text { Matière azotée/matière grasse ........ } r=-0,89 \\
& \text { Matière azotée/calcium ............ } \quad r=-0,96 \\
& \text { Lactose/sodium }+ \text { potassium......... } \quad r=+0,97 \\
& \text { Phosphore/calcium .............. } r=+0,98
\end{aligned}
$$

Toutes ces corrélations sont significatives au seuil $\mathrm{P}=0, \mathrm{or}$.

Dans l'ensemble, comme pour le lait de Truie, il y a des liaisons étroites entre l'évolution des principaux constituants et ceux-ci ne varient donc pas de façon indépendante. On notera en particulier, qu'il existe une liaison inverse très nette entre la teneur en matière azotée et la teneur des autres constituants organiques et même de certains minéraux ( $\mathrm{P}, \mathrm{Ca})$. La liaison inverse entre le lactose et les métaux alcalins $(\mathrm{Na}+\mathrm{K})$ n'est pas non plus sans intérêt.

\section{b) Relations inter-individuelles}

Le tableau 8 rapporte les valeurs trouvées pour les coefficients de corrélation entre les constituants des sécrétions individuelles à un même stade de prélèvement. Les calculs ont été faits à 3 moments différents et les résultats sont comparés à ceux que nous avions trouvés antérieurement sur l'ensemble de la lactation. 
TABLEAU 8

Corrélations inter-individuelles entre les constituants de la sécrétion lactée de la Truie

\begin{tabular}{|c|c|c|c|c|}
\hline Stade de lactation & Mise bas & $24 \mathrm{~h}$ & $48 \mathrm{~h}$ & $\begin{array}{l}\text { Ensemble de la } \\
\text { lactation (1) }\end{array}$ \\
\hline $\begin{array}{l}\text { Lactose/matière azotée.... } \\
\text { Lactose/matière grasse ... } \\
\text { Matière grasse/matière azo } \\
\text { tée ................... } \\
\text { Lactose/sodium .......... } \\
\text { Matière azotée/calcium.... }\end{array}$ & $\begin{array}{l}-0,39 \\
+0,22 \\
-0,30 \\
-0,11 \\
-0,20\end{array}$ & $\begin{aligned}- & 0,5,3^{*} \\
& 0,00 \\
- & 0,23 \\
- & 0,66 * \\
- & 0,26 i\end{aligned}$ & $\begin{array}{l}-0,67^{*} \\
-0,54^{*} \\
+0,51 \\
-0,42 \\
-0,10\end{array}$ & $\begin{array}{l}-0,55^{* *} \\
-0,54^{* *} \\
+0,42 * * \\
-\end{array}$ \\
\hline
\end{tabular}

(1) E. SALmon-Legagneur, $1961 ; \quad$ * significatif à $\mathrm{P}=0,05 ; \quad$ ** significatif à $\mathrm{P}=0,01$.

Vu le faible nombre de données, ces coefficients de corrélations sont peu significatifs, mais ils apportent cependant quelques indications : on retrouve en général au début de la lactation, les mêmes liaisons que celles que nous avions trouvées au cours de la lactation proprement dite.

Mais ces liaisons sont beaucoup moins nettes et souvent sans signification pour les tout premiers stades (mise bas, 24 heures), ce qui semble montrer une certaine évolution dans les rapports entre constituants.

Parmi les liaisons les plus constantes, il faut surtout retenir les corrélations inverses entre le lactose et les matières azotées et entre le lactose et le sodium.

\section{DISCUSSION}

\section{A. - Composition du colostrum}

Il y a peu de remarques à faire en ce qui concerne la composition moyenne du colostrum. Les valeurs que nous avons trouvées sont assez proches de celles rapportées par les autres auteurs (tableau I), compte tenu du fait que les dates de prélèvements ne coïncident pas toujours. Ia grande variabilité inđividuelle des teneurs en certains constituants explique également une partie des différences, notamment celles qui ont trait aux taux de matière grasse et de calcium.

\section{B. - EVOlution DES PRINCIPAUX CONSTituANTS}

Cette évolution offre plusieurs points à considérer :

Tout d'abord elle paraît suivre pour chaque constituant des lois apparemment bien définies, mais dont l'étude nous entraînerait malheureusement trop loin. On retiendra toutefois l'allure très particulière de l'évolution du taux de matière sèche dont la courbe paraît s'apparenter à une fonction du $3^{\mathrm{e}}$ degré et que l'on trouve déjà dans le travail de Travnicerk (I960). 
A côté, l'évolution du taux de matière azotée paraît très classique, alors que celle du taux de matière grasse paraît caractéristique de l'espèce porcine.

La plupart de ces courbes présentent toutefois un trait commun, qui est la rapidité de $1^{\prime}$ 'évolution entre la $6^{\mathrm{e}}$ et la $3^{\mathrm{e}}$ heure après la parturition. Certaines courbes présentent un point d'inflexion (matière grasse, calcium, phosphore, sodium) et d'autres un changement de pente important (matière azotée), situés en général vers la $24^{\mathrm{e}}$ heure.

Il est vraisemblable que cette phase d'évolution accélérée correspond à 1'augmentation de l'activité sécrétoire générale de la mamelle et, en particulier, à l'augmentation du volume de la sécrétion. Peut-être faut-il voir dans cette courte période les limites de la " période colostrale " qui sont précisément fixées, selon HoudiniERE (I944), par la "vitesse de variation des constituants ". Dans ce cas, on pourrait admettre que la sécrétion du colostrum n'excède pas 48 heures chez la Truie.

Mais c'est surtout la comparaison avec les autres espèces qui paraît intéressante. Si l'on s'en tient aux espèces dont la sécrétion mammaire est la mieux connue, c'est-à-dire essentiellement les espèces bovine, ovine et caprine, on constate une similitude d'évolution avec les Truies pour les teneurs en lactose, en matière azotée, en sodium et en potassium (SAVINI, I927 - PARRISH, et al., I95o-BERGMAN, I937 - Perrin, I958 - Sugai et Kuramoto, i959- Journet et Jarrige, i96o). Par contre, les évolutions sont très différentes pour les lipides, le calcium et le phosphore.

Peut-on en déduire que le mécanisme de la sécrétion de certains constituants est différent chez la Truie?

Constatons tout d'abord qu'il existe d'autres espèces qui se comportent comme la Truie, par exemple la Jument : LINTON (I93I) et FLADE (I955) ont en effet montré que le colostrum de Jument s'enrichissait en lipides pendant les premières heures et que le taux maximum de lipides n'était atteint qu'au bout de 48 heures. Chez la Femme également, la sécrétion s'enrichit en matière grasse pendant les premiers jours de lactation (DAvis, r953). Enfin, même chez la Vache, il n'est pas rare que la teneur en matière grasse du colostrum soit inférieure à celle du lait des premiers jours (Journet et JARrige, rg6o). Par contre, chez la Ratte (Glass, I957), la Chienne (ANderson et al., I940) et même chez une espèce sauvage voisine du Porc, le Pécari à collier, pecari tajacu (SoLws et al., I96I) la teneur du colostrum en lipides paraît plus élevée que celle du lait. Mais on a vu que chez le Porc la teneur en matière grasse de la sécrétion montait très rapidement dans les heures qui suivaient la mise bas et il a pu en être ainsi chez ces dernières espèces.

Nous en venons donc à penser que ce qui pourrait varier entre ces différentes espèces serait moins le sens de l'évolution des constituants que la chronologie de cette évolution. Cette hypothèse semble confirmée en particulier par les études sur la composition de la sécrétion antepartum de la Vache. PIETRE (I934) avait montré, en effet, l'existence chez cette espèce d'un " miel colostral " pauvre en matière grasse et en calcium, mais riche en sodium, apparaissant dans la mamelle dès le $5^{\mathbf{e}}$ mois. En outre, les études de RowLAND et al., (I953) sur la traite antepartum montrent bien qu'il y a chez la Vache une évolution de la composition de la sécrétion totut à fait comparable à celle que nous signalons chez la Truie après la mise bas (fig. 9 et Io). Il y aurait en définitive un décalage d'une dizaine de jours entre ces deux espèces. Tout semblerait se passer comme si la phase de sécrétion précolostrale était retardée et réduite chez la Truie. 


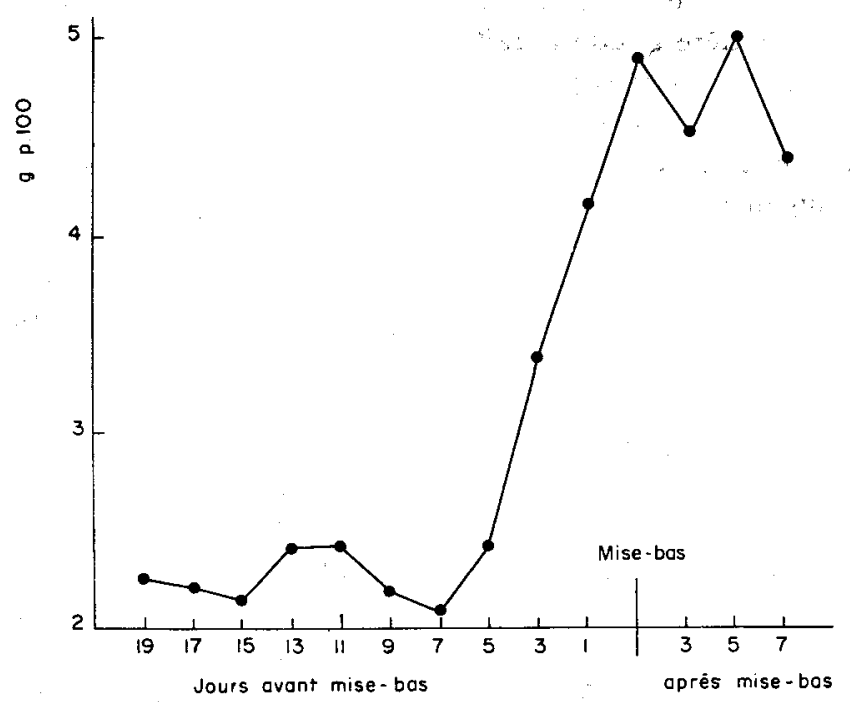

Fig. 9. - Évolution du taux butyreux de la sécrélion lactée pre et post partum de la vache (selon Rowland et al., 1953)

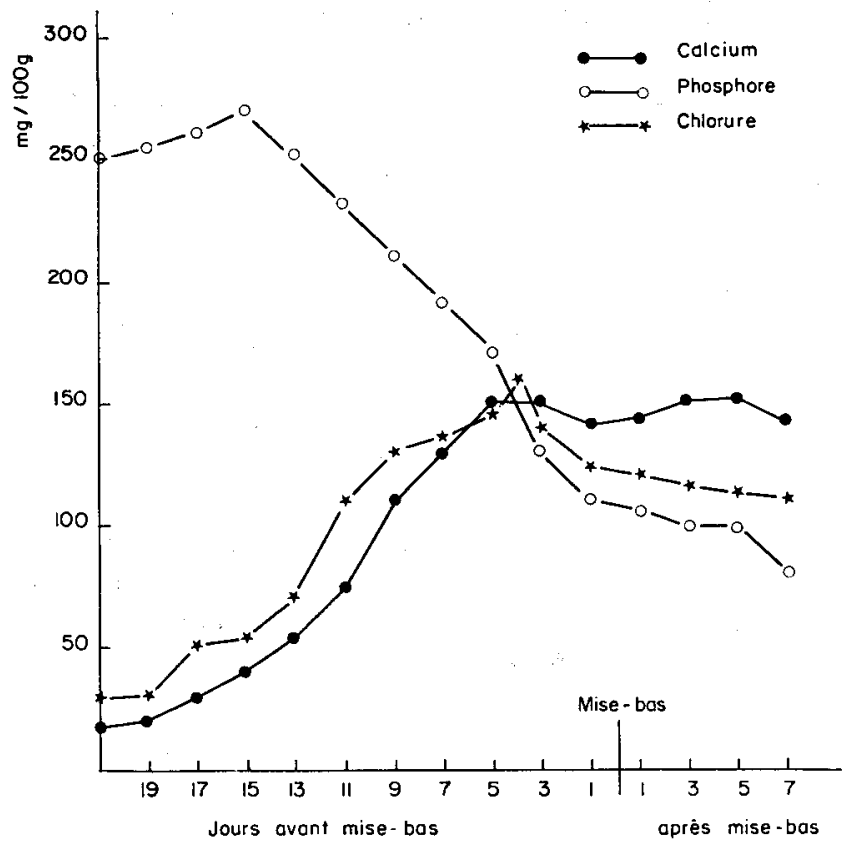

FIG. 1o. - Évolution des teneurs en minéraux de la sécrétion lactée pre et post partum de la vache (selon RowLAND et al., 1953) 
Ceci a été l'objet d'une autre confirmation. En dépit du fait que la mamelle est apte à sécréter bien avant la fin de la gestation (MAYER, I96I), on sait maintenant que la Truie est une des espèces chez laquelle ce phénomène est le plus tardif. Selon Gross et al. (r957), la sécrétion n'apparaît que 24 heures avant le part chez la Truie, alors qu'elle apparaît 60 jours avant chez la Chèvre et 5 mois avant chez la Vache (TURNER, I952).

Tout semble donc concourir à expliquer la différence d'évolution de la composition de la sécrétion mammaire de la Truie et de celle des autres espèces. Contrairement à ce que l'on a parfois avancé, la Truie ne fait pas exception aux mécanismes habituels de la sécrétion lactée; ce sont seulement les modalités qui diffèrent.

\section{C. - Fractions azotées}

En ce qui concerne la teneur du colostrum de Truie en azote de différente nature, nos résultats sont sensiblement en accord avec ceux de SHEFFy et al. (r952) et ceux de Travnicek (I960), les seuls que nous ayons pu trouver dans la littérature. Ils sont également comparables à ceux que BERGMAN et Turner (I937) trouvent chez la Chèvre et à ceux que RowLAND et al. (I953) indiquent pour le " colostrum normal » de Vache, à une exception près : l'azote non protéique, qui est sensiblement plus élevé chez la 'Truie.

Par contre, lorsque l'on poursuit la comparaison entre espèces sur l'évolution de la répartition des fractions azotées, on trouve rapidement quelques différences. Par exemple, notre travail, confirmé par celui de TravnicEK sur ce point, montre que la caséine semble se stabiliser autour de 50 à 55 p. Ioo de l'azote total au bout de 3 ou 4 jours.

Chez la Vache, ou chez la Chèvre, l'évolution est à la fois plus rapide et plus importante puisque dès le $2^{\mathrm{e}}$ jour de lactation la caséine représente plus de $70 \mathrm{p}$. Ioo de l'azote total (Journet et JARrige, I960 - BERGMan et TURner, I937). Il semblerait donc que l'aptitude à fabriquer de la caséine soit moins élevée chez la Truie que chez les Ruminants. L'hypothèse d'une compétition entre les précurseurs de la caséine et la $\beta$-lactoglobuline n'est pas non plus à écarter (BARRY, I96I).

Enfin, il n'est pas sans intérêt de noter que la fraction non protéique du colostrum de Truie augmente très sensiblement à la fois en valeur absolue et en valeur relative (elle représente 9 p. Ioo au bout de 3 jours), ce qui n'est pas le cas chez la Vache (PARRISH, I948). Comme cette fraction est constituée en particulier par les déchets du métabolisme azoté (SHEFFy et al., I952), il n'est pas impossible que chez la Truie en lactation une part croissante des matériaux azotés d'origine alimentaire ou tissulaire soit dégradée et utilisée à d'autres fins que la protéinogenèse, par exemple à des fins énergétiques, ou à la gluconéogenèse (FOL, I947). Ceci supposerait, l'existence d'un " cycle de l'urée " dans la mamelle de la Truie, ce qui, avouons-le, n'a pu être démontré jusqu'à présent.

\section{D. - RELATIONS ENTRE CONSTITUANTS}

L'existence d'une corrélation, même de coefficient très élevé, entre deux variables ne signifie pas forcément qu'il y ait une liaison fonctionnelle entre ces variables. Le fait que les taux de la plupart des constituants de la sécrétion lactée de la Truie 
soient liés par des corrélations intra ou interindividuelles ne prouve donc pas forcément qu'il y ait dépendance, mais peut-être seulement variations concomitantes. Aussi ne discuterons-nous ici que les relations qui ont fait l'objet d'hypothèses antérieures.

La plus intéressante a trait au lactose et à la matière azotée. En effet, la corrélation négative entre les teneurs de ces deux constituants pour une même truie à différents stades de prélèvement, se double d'une autre corrélation négative entre individus. Autrement dit, plus la sécrétion est riche en matière azotée et moins elle contient de lactose, quel que soit le moment. Il n'en est pas de même pour les autres constituants de la matière organique; par exemple, la matière azotée et la matière grasse varient en sens inverse au début de la lactation, mais au bout de 48 heures il n'en est plus ainsi. Cette situation particulière du lactose et de l'azote nous avait conduit à envisager, après LoDGE (I959), une possibilité de concurrence entre ces deux constituants, autrement dit à reprendre 1'hypothèse de la gluconéogenèse à partir des précurseurs des protéines, émise par Graham (I937).

Cette hypothèse reste donc valable et trouve un appui supplémentaire dans l'augmentation, que nous avons signalée, de l'azote non protéique de la sécrétion parallèlement à celle du taux de lactose.

On a aussi invoqué le rôle complémentaire que le lactose et le sodium pourraient jouer dans le maintien de la pression osmotique de la sécrétion lactée (Rook et WoOD, I958 - Denamur, I96I). Selon cette hypothèse, la sécrétion des ions $\mathrm{Na}^{+}$réglerait le métabolisme de l'eau dans la mamelle et le taux de lactose se réajusterait pour assurer l'isotonicité de la sécrétion. Rien ne s'oppose ici à la validité de cette hypothèse, puisque nous avons trouvé chez la Truie une corrélation négative entre les teneurs individuelles en ces constituants et qu'au cours de la lactation la richesse en sodium et potassium diminue lorsque la teneur en lactose augmente. On peut, en outre, tirer argument du fait que la concentration en lactose augmente, pendant la période considérée, à peu près Io fois plus vite que ne baisse celle $d u$ sodium et du potassium, ce qui correspond sensiblement au rapport des activités osmotiques.

L'évolution en sens inverse des teneurs en phosphore et en calcium d'une part et de la teneur en caséine d'autre part, est par contre plus surprenante. En effet, il est bien connu que la caséine existe normalement dans le lait sous forme d'un complexe de caséinate de calcium et de phosphate tricalcique et que, de ce fait, la majeure partie du calcium et du phosphore de la sécrétion lui est étroitement associée. Or, chez la Truie, le rapport $\mathrm{Ca} / \mathrm{N}$ caséine varie largement pendant la période considérée $(0,08$ à 0,36$)$. Ceci peut indiquer que les possibilités de fixation du calcium par la caséine ne sont pas constantes. Par comparaison avec les valeurs trouvées pour la Vache, il y aurait un déficit de calcium dans le colostrum de Truie et un léger excès dans la sécrétion ultérieure. Comme parallèlement le rapport calcium phosphore s'accroît largement dans le lait (il passe de 0,6 à $\mathbf{I}, 2$ en quatre jours), on peut émettre l'hypothèse d'une augmentation de la proportion de phosphate tricalcique associé à la caséine (en admettant que le phosphore organique et le calcium du caséinate demeurent en proportions bien définies) jusqu'à la formation du complexe caséinique définitif du lait normal. On peut aussi penser à une modification des teneurs des autres combinaisons du calcium (citrate, chlorure). 


\section{CONCLUSION}

L'évolution de la composition chimique de la sécrétion des premiers jours de lactation chez la Truie nous a permis de constater :

Io Que certains constituants varient dans une proportion très importante et avec une grande rapidité. C'est le cas de l'azote total, des matières grasses et du calcium. De ce fait, certains phénomènes, comme la présence d'un maximum pour la teneur en matière grasse atı bout de 36 heures (avec un minimum correspondant pour la teneur en eau) ont pu jusqu'à présent passer parfois inaperçus. Les autres constituants de la sécrétion varient également, mais d'une façon moins importante et plus classique

$2^{\circ}$ Que, toutefois, les différences avec l'évolution des sécrétions lactées d'autres espèces s'expliquent aisément si l'on admet que le déclenchement de la lactogenèse n'a pas lieu au même moment par rapport à la parturition chez ces espèces. Par exemple, la sécrétion débute plus tôt chez les Ruminants que chez la Truie. Ainsi, lès contradictions que l'on note pour l'évolution des teneurs en matière grasse ou en certains minéraux ne sont qu'apparentes, car les mêmes variations se retrouvent dans le temps.

$3^{\circ}$ Que les différentes fractions azotées de la sécrétion, à l'exception peut-être de la fraction non protéique, suivent une évolution analogue chez la Truie et chez les autres espèces. L'effondrement du taux de matières azotées est dû principalement aux protéines du sérum (albumines, globulines, protéose peptones). Par comparaison, le taux relatif de caséine tend à remonter (il passe de 26 à $55 \mathrm{p}$. Ioo), mais il n'atteint pas une valeur aussi élevée chez la Truie que chez les bovins.

$4^{\circ}$ Que les secrétions de certains constituants peuvent ne pas se faire de façon indépendante. Notamment, l'existence de corrélations négatives élevées, jointes à la variation de l'azote non protéique, accréditent l'hypothèse d'une gluconéogenèse partielle, peut être plus importante chez la Truie. Par contre, l'évolution de la teneur en calcium indépendante de celle de l'a zote et partiellement de celle du phosphore peut prêter à différentes interprétations.

\section{Reçu pour publication en juillet 1962}

\section{SUMMARY}

THE CHEMICAL COMPOSITION OF SOW'S COLOSTRUM

The study of variations in water, lactose, fat total nitrogen and nitrogenous fractions, $\mathbf{P}, \mathrm{Ca}$, $\mathrm{K}, \mathrm{Na}, \mathrm{Mg}$ contents of milk during the first four days of lactation has been carried out with I6 sows (I92 samples).

Average results for each constituent are shown in tables 2-7 and figures I-8.

Besides the well known trends of several constituents (lactose, nitrogen, $\mathrm{K}, \mathrm{Na}$ ), the most striking features are the changes in fat percentage, which shows a maximum towards 36 hours and the calcium content, which sharply increases throughout the period. These phenomena however may be explained by a difference between sows and other species in the initiation of the mammary secretion. 
In the course of the period, the serum protein strongly decreases, whereas the non protein nitrogen slightly increases. The relative casein percentage increases to $55 \mathrm{p}$. Ioo.

Relationships between constituents are discussed, namely those between lactose, nitrogen and sodium. They seem to show the importance of lactose secretion in the regulation of osmotic pressure and to provide a new argument for the hypothesis of glucose synthesis from proteins in the sow.

\section{RÉFÉRENCES BIBLIOGRAPHIQUES}

Anderson H. D., Johnson B. C., Arnold A., 1940. The composition of dog's milk. Amer. J. Physiol., 129, $63^{-}-635$.

Aschaffenburg R., Drewry J., 1959. New procedure for the routine determination of the various non-casein proteins of milk. $X V$ Int. Dairy Congress, N. I. R. D., paper I631-I637.

Barry J. M., 196r. Protein metabolism, in Kon S. K. et Cowie A. T., Milk. Vol. 1,383-419, Acad. Press, N. Y.

Berge J., Indrebo T., I953. Milk production by sows. Medl. Norges Landbrukshogsk., 33, $389-423$. Bergman A. J., Turner C. W., I937. The composition of the colostrum of the dairy goat. J. Dairy Sci., 20, 37-45.

Bowland J. P., Grummer R. H., Phililps P. H., Bohstedt G., I949. Effect of plane of nutrition on the composition of sow's colostrum and milk. J. Anim. Sci., 8, 199-206.

Braude R., Coates M. E., Henry K. M., Kon S. K., 1947. A study of the composition of sow's milk. Brit. J. Nutrit., 1, 64 .

Cross B. A., Godwin R. F., Silver I. A., 1958. A histological and functional study of the mammary gland in normal and agalactic sows. J. Endocrin., 17, $63-66$.

Davis J. G., Mac Donald F. J., I953. Richmond Dairy Chemistry, 663 pp. Griffin co. London.

Denamur R., ig6r. (Communication personnelle).

Flade E., 1955. Milchleistung and Milchqualität bei Stutten. Tiersucht., 9, 38r-383.

Folley S. J., Greebaum A. L., i947. Changes in arginase and alkaline phosphatase contents of the mammary gland. Biochem. J., 41, 26. .

GLASS R. L., 1957. Chemical, physical, biological studies of rat's milk and its components. Diss. Abstr., 17, $494-495$.

GRAIIAM W. R., 1937. The production of urea in the mammary gland. J. Biol. Chem., 120, 29-33.

Gueguen L., Salmon-Legagneur E., 1959. La composition du lait de truie : variations des teneurs en quelques éléments minéraux (P, Ca, K, Na, Mg). C. R. Acad. Sci. Paris, 249, 784-786.

Gueguen L., Journet M., i96r. Les variations de la composition du lait de vache. Ann. minérale Biol. Anim. Bioch. Biophys., 1, 305-310.

Houdiniere A., i 944. Le colostrum de vache. Le Lait, 24, I08-I39.

Hughes H. E., Hart H. G., I935. Production and composition of sow's milk. J. Nutrit., 9, 3II-322.

Journet M., Jarrige R., i960. Evolution de la sécrétion des matières grasses, des matières azotées et du lactose au cours du premier mois de lactation. Ann. Zootech., 9, I33-I55.

Linton R. G., I931. The composition of mare milk. J. Agric. Sci., 21, 669-688.

LENKEIT W., 1953. Einführung in die Ernährungsphysiologie der Haustiere, Stuttgart.

LODGE G. A., I959. The composition of sow's milk during lactation with particular reference to the relationship between protein and lactose. J. Dairy Res., 26, I34-г 39 .

Mayer G., KLein M., I961. Histology and cytology of the mammary gland, in Kon S. K. et Cowie A. T., Milk, vol. 1, 47-126, Acad. Press, N.Y.

Niwa T., Ito S., Yokoyama A., Otsuka M., 195r. Studies on the milk secretion of sow, on the habits of nursing, milk yield and constituents of milk of the sow. Bul. Inst. Agric. Sci. Japan, G, 1, I35-I 50.

Ostertag R., ZunTs N., igo8. Untersuchungen über die milchsekretion des Schweines und die Ernährung der Ferkel. Landwirtschaf. Jahrbïcher $B d, \mathbf{3 7}, 5201$.

Parrish D. B., Wise G. H., Hughes J. S., AtKeson F. W., r948. Properties of the colostrum of the dairy cow. II. Effect of prepartal rations upon the nitrogenous constituants. J. Dairy Sci., 31, 889-895.

Parrish D. B., Wise G. H., Hughes J. S., Atkeson F. W., i950. Properties of the colostrum of the dairy cow. V. Yield, specific gravity and concentration of totals solids and its various components of colostrum and early milk. J. Dairy Sci., 33, 457-465.

PERrin D. R., I955. The chemical composition of the colostrum and milk of the sow. J. Dairy Res., 22, I03-107.

PERRIN D. R., I958. The chemical composition of the colostrum and milk of the ewe. J. Dairy Res., 25, $7^{\circ-74}$. 
Pietre M., 1934. Phénomènes physicochimiques accompagnant lentraînement physiologique de la mamelle chez les femelles primipares. C.R. Acad. Sci. Paris, 198, I454-1456.

Rook J. A., Woon M., 1958. Interrelationships of the concentration of sodium, potassium, lactose and water in milk. Nature, 181, I $284-1285$.

Rowland S. J., I938. The determination of the protein distribution in milk. J. Dairy Sci., 9, 30-46.

Rowland S. J., RoY J. H., Sears H. J., Thompson S. Y., 1953. The effect of prepartum milking on the composition of prepartum and postpartum secretions of the cow. J. Dairy Res., 20, 16-28.

SAvini E., 1927. Chimica ed analisi del latte e dei lattini. Hoepti ed. Milan.

SALMON-Legagneur E., 1959. La composition du lait de truie : premières observations sur quelques facteurs de variation. Ann. Zootech., 8, 93-II 2 .

Salmon-Legagneur E., 196r. La composition du lait de truie : relations entre les variations des teneurs du lactose et des autres constituants. Ann. Biol. Anim. Bioch. Byophys., 1, 295-303.

Shahani K. M., Sommer H. H., I95I. The protein and non protein nitrogen fractions in milk. J. Dairy Sci., 34, 1003-1013.

Sheffy B. E., Shahani K. M., Grummer R. H., Phillips P. H., Sommer H. H., I952. Nitrogen constituants of sow's milk as affected by rations and stage of lactation. J. Nutrit., 48, I03-1 I3.

Sowls L. K., Smith V. R., Jenness R., Rehger E., ig6r. Chemical composition and physical properties of the milk of the collared peccary. J. Mammal., 42, 245-25r.

Sugai K., Kuramoto K., I959. Milk secretion studies with corriedales ewes. II. On the composition of corriedale ewe's milk. Sci. Rep. Agric. Okayoma Univ., no 13, 69-73.

TravniceK J., ig60. La composition chimique du colostrum et du lait de truie Edebschwein (Tchèque). Zivocisna Vyroba., 5, 497-5I2.

TURner W., 1952. The mammary gland. Lucas Brothers Publ., Columbia.

Von Gohren T., I865. Analyse der Schweinemilch. Ldw Vers. Sta. Bd. 7.

Waite R., White J., Robertson A., I956. Variations in the chemical composition of milk I. - Effect of stage of lactation, season, age of cows. J. Dairy Res., 23, 65-8r. 